\title{
Pharmacist-led influenza vaccination services in residential aged care homes: a pilot study
}

\begin{abstract}
Objectives: The feasibility of pharmacist-led influenza vaccination services in residential aged care homes has not previously been studied. The primary objective of this pilot study was to evaluate this feasibility. The secondary objective was to assess the effect on employee vaccination rates.
\end{abstract}

Methods: An in-house pharmacist-led vaccination service was implemented at a single site in 2017. De-identified employee influenza vaccination records at this site for 2016 and 2017 were compared to assess the change in vaccination rate.

Results: The residential care pharmacist administered $37 \%$ of all influenza vaccinations to employees ( $n=78)$ in 2017. Between 2016 and 2017 there was a significant improvement in the employee vaccination rate at the site $(46.5 \%$ vs. $69.2 \%, \mathrm{p}<0.01)$.

Conclusion: It is feasible for pharmacists to administer influenza vaccinations to employees in residential aged care. The flexibility of an in-house pharmacist improves accessibility and can therefore promote uptake of influenza vaccination by employees.

Keywords Aged care, employees, influenza, nursing homes, pharmacists, preventive programs, vaccination.

\section{Impact statement}

The feasibility of pharmacist-led influenza vaccination services in residential aged care homes has not previously been reported. Findings of this pilot study indicate that these services are feasible and can significantly improve the uptake of influenza vaccination by employees, warranting further investigation as a potential national strategy to address influenza-related morbidity and mortality in residential aged care homes. 


\section{Introduction}

Influenza is the leading cause of vaccine-preventable death and hospitalisations due to notifiable communicable disease in Australia [1]. Older adults living in residential aged care homes (RACHs) are particularly susceptible to influenza-associated morbidity and mortality due to increased frailty, the presence of multiple chronic medical conditions, and impaired response to anti-viral drug therapy [2]. The annual number of influenza-related deaths in Australia was the highest ever recorded in 2017, with $91 \%$ of influenza-related deaths recorded in people aged over 65 years [3]. RACHs are closed communities which promote the rapid transmission of the influenza virus between residents, increasing the risk of clinical infection [4]. Furthermore, RACH residents are more likely to require hospitalisation due to influenza than individuals residing in the community [5].

The Australian National Health and Medical Research Council recommends annual influenza vaccination as the single most important intervention for preventing influenza [6]. RACH employees work in close contact with multiple residents daily and are an important source of transmission if not vaccinated $[4,7,8]$. Annual influenza vaccination is recommended for all RACH employees, due to their close interaction with older, frail residents [8]. RACHs with an employee vaccination rate above $75 \%$ report lower frequencies of influenza outbreak compared with homes with lower rates of vaccine uptake amongst employees [9]. A vaccination rate of $95 \%$ of all residents and employees prior to the influenza season is recommended to induce herd immunity and protect residents [9].

Annual influenza vaccination is not currently mandatory for $\mathrm{RACH}$ employees in Australia and uptake is at the discretion of the individual [9]. Consequently, the RACH employee influenza vaccination rates are suboptimal, with less than $4 \%$ of RACHs reporting that they have achieved the recommended vaccination rate of 95\% [9]. Approximately $43 \%$ of RACHs have influenza vaccination rates at less than 50\% [9]. Employee vaccination rates within RACHs in the Australian Capital Territory (ACT) are particularly low, with reported vaccination rates as low as $28 \%$ [4]. Reasons cited by RACH employees for not receiving annual influenza vaccination include: concerns about vaccine side effects, perceived lack of vaccine efficacy or necessity, vaccination not recommended by the employer/organisation, affordability, inconvenience, and accessibility $[4,8,10]$. 
Some of these barriers may be circumvented through flexible on-site employee vaccination programs offered at no cost by RACH organisations. Approximately $86 \%$ of Australian RACHs report having an established program which provides vaccines to employees [9]. However, many on-site programs are outsourced to external providers who offer a limited number of fixed-duration vaccination clinics for employees, restricting opportunities for shift workers. Given the largely casualised and part-time nature of the RACH workforce in Australia [11], offering a vaccination program with fixed availability for a limited duration may be unsatisfactory for employees working outside of usual business hours, who might also find it difficult to visit their general practitioner (GP). Almost $10 \%$ of RACHs do not offer any on-site vaccinations but encourage employees to obtain the vaccine from their GP [9].

The Australian population is aging and demand for RACH placements is increasing [12]. Therefore, identifying strategies for optimising vaccination rates for RACH employees to protect residents from influenza is of growing importance. Pharmacists have been permitted to vaccinate people against influenza in the Australian community since 2015 [13]. The rationale for this initiative was to improve accessibility for members of the community who have difficulty accessing vaccination through their GP or employer, as pharmacies tend to have extended business hours [13]. Similarly, having an on-site pharmacist within RACHs, available to administer influenza vaccinations to employees as a component of their role, may increase flexibility and convenience for the provision of vaccination. Further, pharmacists are appropriately qualified to educate employees on the risks and benefits of vaccination including potential side effects, and the importance of receiving vaccination to protect residents and themselves.

To date, the effect of pharmacist-led vaccination services on RACH employee vaccination rates has not been explored. The primary objective of this pilot study was to evaluate the feasibility of an in-house influenza vaccination service provided by a pharmacist. The secondary objective was to evaluate the effect of the service on RACH employee influenza vaccination rates.

\section{Methods}

The pilot study was part of a broader trial exploring the feasibility of integrating a residential care pharmacist (RCP) into an established team within a RACH located in the ACT [14]. The 
RCP provided influenza vaccinations to both employees and residents as part of the broader trial addressing multiple quality use of medicines components.

A vaccination-accredited RCP was piloted for six months from April 20th, 2017 to October 13th, 2017. The RCP was employed part-time, two consecutive days per week (0.4 full time equivalent) for the duration of this period. Employee influenza vaccination registers for 2016 and 2017 were maintained by the clinical care manager, documenting employees who had received a vaccine on-site or provided evidence from their external provider, such as a GP. The registers included all full-time, part-time and casual nursing, care, administration, cleaning, and catering employees at the RACH. Deidentified data were provided to the principal investigator for analysis at the end of the RCP trial period. The RCP had documented the number of vaccinations provided to employees and the time spent performing this activity on each occasion. The study site had a total of 71 employees in 2016 and 78 employees in 2017. Self-reported data from $21 \mathrm{RACHs}$ with known vaccination rates in the ACT was provided by the Australian Aged Care Quality Agency [15].

Fisher's exact test was used to compare employee vaccination rates in 2016 and 2017 at the study site. A one-sample proportions test compared the vaccination rate at the study site in 2017 with the state average self-reported rate of vaccinations in 2017. All analyses were conducted using SPSS Statistics version 22.0 (IBM, Armonk, NY, USA) and p-values of $<0.05$ were considered statistically significant. This research was approved by the University of Canberra Human Research Ethics Committee (HREC 16-244).

\section{Results}

Of the total number of 78 employees at the RACH in 2017, 54 (69.2\%) received an influenza vaccine. The employee influenza vaccination rate improved significantly from $46.5 \%$ in 2016 (Fisher's exact $\mathrm{p}<0.01$ ). The RCP spent a total of 3.4 hours administering influenza vaccines to $20(37 \%)$ employees in 2017 . The difference between the employee vaccination rate at the study site (69.2\%) and the ACT state average in 2017 (54.2\%) was significant $(\mathrm{z}=2.66$, $\mathrm{p}<0.01)[15]$.

No adverse events were recorded in association with the pharmacist-administered vaccines. The RCP also recorded administration of vaccines to two residents at the study site who were unable to obtain the vaccine from their GP, and four employees from a separate $\mathrm{RACH}$ in nearby proximity to the study site. 


\section{Discussion}

This is the first Australian study to investigate an in-house pharmacist-led influenza vaccination service in residential aged care. The findings indicate that it is feasible to include an in-house pharmacist in RACHs, who can provide a flexible option to administer influenza vaccinations to fellow employees. While registered nurses working within the RACH could theoretically have administered influenza vaccines to fellow employees, it was noted that they were fully occupied on their primary duty of providing time-critical clinical care activities directed at residents.

The pharmacist-led vaccination service contributed to an increased vaccination rate of $22.7 \%$, resulting in a vaccination rate of $69.2 \%$ in 2017 . This compared favourably and significantly with the average self-reported rate of $54.2 \%$ for ACT RACHs. The increased uptake of influenza vaccination by employees was facilitated by the implementation of an inhouse program with the capacity to administer ad-hoc vaccinations, rather than at fixed clinic or appointment times available through external providers. Convenience and accessibility are two leading barriers to RACH employee uptake of influenza vaccination cited in the literature $[4,8,10]$.

This was a single-site pilot study, without a control site. Another limitation was the part-time nature of the RCP role, who was only on-site at the RACH two consecutive days per week. This limited the accessibility of vaccination by the RCP for employees working exclusively on weekends, evening shifts and part-time on days where the RCP was not present. Further, the timing of influenza vaccination is recommended to commence in March and April once the annual vaccine is available and prior to peak influenza season starting from June [2]. The RCP did not commence employment until late April, limiting opportunities for providing employee education and promotion of the service to overcome perceived lack of vaccine efficacy or necessity as a barrier to uptake $[4,8,10]$. These issues may have contributed to the final employee vaccination rate in 2017 still being below the recommended vaccination rate of $95 \%[9]$.

Additionally, the acceptability of this service from the perspective of the RACH employees was not formally evaluated. Finally, this service was feasible under ACT legislation where pharmacists are not restricted to administering influenza vaccinations within a 
community pharmacy [16]. Legislation changes would be required nationally for this model to be successful in other states and territories.

\section{Conclusion}

This pilot study demonstrated that it is feasible to include pharmacists as part of in-house influenza vaccination programs in RACHs. The implementation of the service significantly increased the uptake of influenza vaccination by $\mathrm{RACH}$ employees. A larger trial, incorporating a comprehensive assessment of acceptability, clinical outcomes and costeffectiveness, is warranted.

Acknowledgements The authors would like to acknowledge Goodwin Aged Care Services and employees, and Mr Richard Thorpe for piloting the role of the residential care pharmacist.

Conflicts of interest TM is an employee of the funding organisation, Goodwin Aged Care Services. All other authors declare no conflict of interest.

Declaration of Sources of Funding Clinical components of this study were funded by Goodwin Aged Care Services. NM is undertaking a $\mathrm{PhD}$ funded by a Commonwealth scholarship: the Australian Government Research Training Program Stipend Scholarship.

Trial registration This study reports findings associated with the secondary outcome listed under a broader trial retrospectively registered under the Australian New Zealand Clinical Trials Register, reference number: ACTRN12617001506381 (registered: 25/10/2017) Universal trial number U1111-1200-3611.

\section{References}

1. Australian Institute of Health and Welfare 2018. Australia's health 2018. Australia's health series no. 16. [online]. Available at: https://www.aihw.gov.au/reports/australias-health/australias-health2018/contents/table-of-contents [accessed 26 June 2018].

2. MacIntyre CR, Menzies R, Kpozehouen E, Chapman M, Travaglia J, Woodward M, et al. Equity in disease prevention: Vaccines for the older adults - a national workshop, Australia 2014. Vaccine. 2016;34(46):5463-9. 
3. National Influenza Surveillance Committee. 2017 Influenza season in Australia. A summary from the National Influenza Survellance Committee [online]. Australian Department of Health. 2017. Available at: http://www.health.gov.au/internet/main/publishing.nsf/Content/ozflu-surveil-2017final.htm [accessed 24 May 2018].

4. Halliday L, Thomson JA, Roberts L, Bowen S, Mead C. Influenza vaccination of staff in aged care facilities in the ACT: How can we improve the uptake of influenza vaccine? Australian and New Zealand Journal of Public Health. 2003;27(1):70-5.

5. Ingarfield SL, Finn JC, Jacobs IG, Gibson NP, Holman CDAJ, Jelinek GA, et al. Use of emergency departments by older people from residential care: A population based study. Age and Ageing. 2009;38(3):314-8.

6. National Health and Medical Research Centre. The Australian Immunisation Handbook 10th Ed. Canberra: NHMRC 2017.

7. Wendelboe AM, Grafe C, McCumber M, Anderson MP. Inducing Herd Immunity against Seasonal Influenza in Long-Term Care Facilities through Employee Vaccination Coverage: A Transmission Dynamics Model. Computational and Mathematical Methods in Medicine. 2015; DOI $10.1155 / 2015 / 178247$.

8. Apenteng BA, Opoku ST. Employee influenza vaccination in residential care facilities. American Journal of Infection Control. 2014;42(3):294-9.

9. Australian Aged Care Quality Agency. Review of infection control practices in residential aged care in Australia.[online]: Available at: https://www.aacqa.gov.au/publications/infectioncontrol/infection-control-influenza [accessed 10 May 2018].

10. Lorenc T, Marshall D, Wright K, Sutcliffe K, Sowden A. Seasonal influenza vaccination of healthcare workers: systematic review of qualitative evidence. BMC health services research. 2017;17(1):732.

11. Australian Government Department of Health. 2016 National Aged Care Workforce Census and Survey - The Aged Care Workforce, 2016. [online]. 2017. Available at: https://agedcare.health.gov.au/sites/g/files/net1426/f/documents/03_2017/nacwcs_final_report_29031 7.pdf [accessed 24 May 2018].

12. Australian Institute of Health and Welfare. Services and places in aged care [online]. Available from: https://gen-agedcaredata.gov.au/Topics/Services-and-places-in-aged-care [accessed 24 May 2018]. 
13. Carroll PR, Hanrahan JR. Current research: A shot in the arm: Pharmacist-administered influenza vaccine in New South Wales. The Australian Journal of Pharmacy. 2017;98(1165):56-9.

14. McDerby N, Naunton M, Shield A, Bail K, Kosari S. Feasibility of Integrating Residential Care Pharmacists into Aged Care Homes to Improve Quality Use of Medicines: Study Protocol for a NonRandomised Controlled Pilot Trial. International Journal of Environmental Research and Public Health. 2018;15(3):499.

15. Australian Aged Care Quality Agency. Data for the Quality Agency's 2017 infection control survey [Data file]. [Accessed 30 August 2018].

16. Medicines, Poisons and Therapeutic Good Regulation 2008, section 352, (Authorisation for pharmacist and intern pharmacist to administer vaccine without prescription - Act, s 37 (1) (b)) [online]. Available at http://www.legislation.act.gov.au/sl/2008-42/current/pdf/2008-42.pdf [accessed June 2018]. 